\title{
I-Wire Heart-on-a-Chip II: Biomechanical analysis of contractile, three-dimensional cardiomyocyte tissue constructs
}

Alison K. Schroer ${ }^{a^{\star}}$, Matthew S. Shotwell ${ }^{\mathrm{b}}$, Veniamin Y. Sidorov ${ }^{\mathrm{a}, \mathrm{c}}$, John P. Wikswo ${ }^{\mathrm{a}, \mathrm{c}, \mathrm{d}, \mathrm{e}}$, W. David Merryman ${ }^{\mathrm{a}^{*}}$

a Department of Biomedical Engineering, Vanderbilt University, Nashville, TN, USA 37235

${ }^{\mathrm{b}}$ Department of Biostatistics, Vanderbilt University, Nashville, TN, USA 37232

${ }^{\mathrm{c}}$ Vanderbilt Institute for Integrative Biosystems Research and Education, Vanderbilt University, Nashville, TN, USA 37235

${ }^{d}$ Department of Molecular Physiology and Biophysics, Vanderbilt University, Nashville, TN, USA 37232

e Department of Physics and Astronomy, Vanderbilt University, Nashville, TN, USA 37235

* Corresponding author, Room 9445D MRB4, 2213 Garland Avenue, Nashville, TN 37212 , USA.

E-mail address: david.merryman@vanderbilt.edu (W. D. Merryman) and alison.k.schroer@vanderbilt.edu (A.K. Schroer) 


\section{Abstract}

This companion study presents the biomechanical analysis of the "I-Wire" platform using a modified Hill model of muscle mechanics that allows for further characterization of construct function and response to perturbation. The I-Wire engineered cardiac tissue construct (ECTC) is a novel experimental platform to investigate cardiac cell mechanics during auxotonic contraction. Whereas passive biomaterials often exhibit nonlinear and dissipative behavior, active tissue equivalents, such as ECTCs, also expend metabolic energy to perform mechanical work that presents additional challenges in quantifying their properties. The I-Wire model uses the passive mechanical response to increasing applied tension to measure the inherent stress and resistance to stretch of the construct before, during, and after treatments. Both blebbistatin and isoproterenol reduced prestress and construct stiffness; however, blebbistatin treatment abolished subsequent force-generating potential while isoproterenol enhanced this property. We demonstrate that the described model can replicate the response of these constructs to intrinsic changes in force-generating potential in response to both increasing frequency of stimulation and decreasing starting length. This analysis provides a useful mathematical model of the I-Wire platform, increases the number of parameters that can be derived from the device, and serves as a demonstration of quantitative characterization of nonlinear, active biomaterials. We anticipate that this quantitative analysis of I-Wire constructs will prove useful for qualifying patient-specific cardiomyocytes and fibroblasts prior to their utilization for cardiac regenerative medicine.

Keywords: cardiac tissue engineering, auxotonic contraction, Hill model muscle mechanics, regeneration 


\section{Introduction}

Three-dimensional engineered cardiac tissue constructs (ECTCs) fill an important gap in understanding cardiomyocyte $(\mathrm{CM})$ function, fibroblasts, and the extracellular matrix $(\mathrm{ECM})$ they produce in a physiologically relevant, in vitro context. ECTCs exemplify the challenges associated with characterizing a nonlinear, dissipative, active biomaterial with a mathematical model. We developed the "I-Wire" ECTC platform to probe the function of CMs while both the applied force and length are changing during auxotonic contraction [1]. This ECTC design better approximates the environment in vivo, since CMs contract against the changing load of ventricular pressure in their native environment. While the I-Wire offers useful insights into CM mechanics and function, the dynamically changing length and force can make analysis more complex than a simple isometric or isotonic contraction. Here we have developed an analysis strategy that permits extraction of different CM and ECTC properties and physiologic responses.

The force-generating capacity of cardiac muscle is inherently dependent on length, passive stiffness, and velocity of contraction [2-4]. A simple model for muscle mechanics that includes damped parallel and series elastic springs was originally proposed by Hill, and it has been used to describe striated muscle mechanics in many contexts [2;5-9]. Although the Hill model has limitations, it has been used and modified in numerous applications, such as predictions of the relevant mechanics involved in heart failure and interventional treatment strategies [10-14]. The objective of this work is to use a modified Hill model to predict changes in CM mechanics in the I-Wire construct to gain insight into clinically relevant mechanical effects, and to demonstrate the detailed characterization of an active tissue equivalent of significant clinical interest. 


\section{Methods}

\subsection{ECTC creation and measurement}

ECTCs were formed and their mechanical response to increasing, perpendicularly applied force was measured as already described [1]. Briefly, CMs were isolated from neonatal rat hearts and embedded in a fibrin matrix in a polydimethylsiloxane (PDMS) mold and allowed to form a 3D cable suspended in media and anchored on conductive wires (Fig. 1A). To create longitudinal tension in the construct, a translatable stage applies transverse force to the construct with a flexible probe, and the deformation of the probe in response to the force applied to the construct allows for precise measurement of both the tensile properties and the developed force in the construct over the course of a contraction (Fig. 2B). Contraction was stimulated by a 5 ms pulse (six times threshold) delivered through the anchoring wires, and probe position was recorded at 200 frames per second. For each contraction condition, seven successive contractions were averaged. The passive and peak developed force, as presented in the companion paper, were calculated as the transverse force exerted on the probe by the construct $\left(F_{P}\right)$ and were proportional to the deflection of the probe from center $\left(\Delta s_{1}\right)$ multiplied by the stiffness of the probe $\left(K_{P}\right)$; (Fig. 1F of [1]) and Eq. (1). The following equations describe how to transform a transverse force applied by the probe by stage displacement to a longitudinal force along the length of the construct $\left(F_{C}\right)$. We start with Hooke's law:

$$
F_{P}=K_{P} \Delta s_{1}
$$

The lateral deflection of the probe tip $\left(\Delta s_{1}\right)$ and the lateral displacement of the construct midpoint $\left(\Delta s_{2}\right)$ sum to the total deflection of the stage $(\Delta s)$ :

$$
\Delta s_{1}=\Delta s-\Delta s_{2}
$$


$\Delta s_{2}$ can be calculated from the extension of the construct $(\Delta L)$ according to the Pythagorean Theorem

$$
\Delta s_{2}=\sqrt{(L+\Delta L)^{2}-L^{2}}=\sqrt{\Delta L(2 L+\Delta L)},
$$

which in turn can be substituted into Eq. (2), and further into Eq. (1) to yield

$$
\begin{aligned}
& \Delta s_{1}=\Delta s-\sqrt{\Delta L(2 L+\Delta L)}, \\
& F_{P}=K_{P}(\Delta s-\sqrt{\Delta L(2 L+\Delta L)}),
\end{aligned}
$$

which describe the transverse force in terms of construct stretch along its length and initial stage displacement. Finally, $F_{P}$ is balanced by the lateral component of force developed along the construct direction in both halves of the construct. Simple trigonometry shows that

$$
F_{C}=\frac{F_{P}(L+\Delta L)}{2 \Delta s_{2}}=\frac{F_{P}(L+\Delta L)}{2 \sqrt{\Delta L(2 L+\Delta L)}},
$$

as derived in the Supplementary Material.

\section{Table 1: Model variables}

\section{Variable}

$\Delta s \quad$ Distance I-Wire frame is moved laterally to apply force with the probe $(\mathrm{m})$

$\Delta s_{1}$

$\Delta s_{2}$

$F_{P}$

$F_{C}$

$L$

$L_{\text {int }}$

$\Delta L$

$\Delta L_{\text {offset }}$

prestress

$K_{M}$

$K_{P}$

$K_{\text {PEam }}$

\section{Description (Units)}

Distance the end of the cantilever probe is deflected $(\mathrm{m})$

$\Delta s-\Delta s_{1}(\mathrm{~m})$

Transverse force delivered by the cantilever probe $(\mathrm{N})$

Longitudinal force within the ECTC in response to $F_{P}(\mathrm{~N})$

Half-length of unstretched I-Wire construct, in practice $3.5 \mathrm{~mm}(\mathrm{~m})$

Intrinsic half-length of the ECTC were its end wires released $(m)$

Change in ECTC half-length as the probe stretches the ECTC $(\mathrm{m})$

Estimated difference between $L$ and $L_{\text {int }}$

Estimated tension in unstretched construct at length $L(\mathrm{~N})$

Steady-state stiffness of muscle construct $\left(\mathrm{Nm}^{-1}\right)$

Cantilever probe spring constant $\left(\mathrm{Nm}^{-1}\right)$

Spring constant of actin/myosin parallel element $\left(\mathrm{Nm}^{-1}\right)$ 


\begin{tabular}{cl}
\hline$K_{S E}$ & Spring constant of series element $\left(\mathrm{Nm}^{-1}\right)$ \\
$K_{P E n}$ & Spring constant of non-actin/myosin parallel element $\left(\mathrm{Nm}^{-1}\right)$ \\
$b$ & Dashpot stiffness $\left(\left(\mathrm{Nm}^{-1} \mathrm{~s}\right)\right)$ (Note data reported in $\mathrm{Nm}^{-1}$ milliseconds) \\
\hline
\end{tabular}

Action potentials in the ECTC were recorded by using floating micropipettes filled with 3-M $\mathrm{KCl}$ [15]. The micropipettes were pulled from borosilicate glass capillaries (WPI, Sarasota, FL, USA) by a micropipette puller (P80/PC, Sutter Instruments, Novato, CA, USA). The tips of the micropipettes were mounted on a platinum wire of $50 \mu \mathrm{m}$ diameter. The reference $\mathrm{Ag} / \mathrm{AgCl}$ electrode (EP8, WPI, Sarasota, FL, USA) was placed in the well next to the PDMS insert. The electrodes were connected with a dual differential electrometer (Duo 773, WPI, Sarasota, FL, USA) and signals were digitized, visualized, and recorded by a digital oscilloscope (TDS5034B, Tektronix, Beaverton, OR, USA). The sampling rate was $25 \mathrm{kHz}$. The recorded data were processed with a Savitzky-Golay digital filter (OriginLab, Northampton, MA, USA). To inhibit contractility, the excitation-contraction uncoupler blebbistatin (Sigma-Aldrich, St. Louis, MO, USA) was applied at a concentration of $6 \mu \mathrm{M}$.

\subsection{Model development}

In recognition of the complexity of cardiac tissue as a biomaterial, we divided the parallel elastic resistance term in the traditional Hill model to differentiate the passive contributions of actin myosin interactions and other passive mechanical linkages, including microtubules, zdiscs, and extracellular matrix. The second passive element $\left(K_{P e n}\right)$ (Fig. 1D) was not responsive to treatment with blebbistatin or isoproterenol. Blebbistatin is a reversible specific inhibitor of the actin-myosin interaction [16]. In particular, it binds to the myosin-ADP- $P_{i}$ complex, impedes phosphate release, and thereby stabilizes the metastable state of myosin [17], whereas isoproterenol is a known $\beta$-adrenergic stimulator that affects both contractility and heart rate [18]. 
When transformed from passive transverse force exerted on the probe to passive tension along the construct length (Eq. (6)), the resultant forces have a linear relationship with respect to the steady-state relative passive length change with a non-zero prestress y-intercept (Fig. 2A,B) as follows:

$$
F_{C}=K_{M}\left(\Delta L+\Delta L_{\text {offset }}\right)=K_{M} \Delta L+\text { prestress }
$$

with $K_{M}$ serving as a lumped parameter of the three elastic elements,

$$
K_{M}=\frac{K_{P E a m}+K_{P E n}+\frac{K_{P E n} K_{P E a m}}{K_{S E}}}{1+\frac{K_{P E a m}}{K_{S E}}} .
$$

This muscle mechanics model is linked to a nonlinear (with respect to $\Delta L$ ) spring (Fig. 1C; right side) that represents the force applied by the probe to the construct (Eq. (9)), which should be equivalent to the value of the force in the construct (Eq. (7)),

$$
F_{C}=\frac{1}{2} K_{P}\left(\frac{\Delta s}{\sqrt{\Delta L(2 L+\Delta L)}}-1\right)(L+\Delta L)
$$

The passive resistance of the muscle was used to estimate model parameters $K_{M}$, prestress, and $\Delta L_{o f f}$ (Fig. 2) for each construct, before and after different perturbations (blebbistatin treatment: $6 \mu \mathrm{M}$; isoproterenol treatment: $1 \mu \mathrm{M}$; the incubation time for blebbistatin and isoproterenol was 10 min; shortening the construct: 20 percent of original length).

The final step in the analysis, described in detail in the Supplementary Material, is to derive the differential equation that relates $\Delta L$ to its rate of change of $\dot{\Delta L}$

$$
\dot{\Delta} L=\frac{\left(1+\frac{K_{P E a m}}{K_{S E}}\right) f_{1}(\Delta L)-K_{M}\left(\Delta L+\Delta L_{o f f s e t}\right)-F_{A}}{b\left(1-\frac{f_{2}(\Delta L)-K_{P E n}}{K_{S E}}\right)}
$$

which can be used to predict the contraction traces by solving it in MATLAB using ode15s. This is the Nashville single-string guitar equation. 


\subsection{Study design statistical analysis}

Five independent constructs were treated with blebbistatin and changes in $K_{M}$ were quantified from the mean of the constructs' passive tension before and after treatment over a range of stage displacements $(0-1300 \mu \mathrm{m})$. Experimental measurements are presented as means $\pm S E M$, and force measurements were compared at each stage position using an unpaired t-test. Seven constructs were treated with isoproterenol, and their passive mechanics and dynamic contraction were fit independently over a range of stage displacements (0-2000 $\mu \mathrm{m})$, which correspond to increasing applied transverse loads.

The passive model parameters were estimated for each construct using linear least squares analysis of the passive force measurements. Active contraction was then simulated in the model by an activation force function originating in the contractile element with a magnitude of around $1.4 \mathrm{mN}$ (within a physiological range for a construct with $0.1 \mathrm{~mm}^{2}$ area [19]) and a duration of $250 \mathrm{~ms}$ (the measured length of an action potential in these constructs [1]). Three active parameters $\left(K_{S E}, K_{\text {PEam }}\right.$, and $\left.b\right)$ were estimated using a nonlinear least squares optimization technique in MATLAB. More information about the model formulation and optimization techniques can be found in the Supplementary Material. The confidence intervals of parameter estimates were approximated using the observed information method [20]. The effects of pharmacologic treatment on the model parameters conserved across constructs were assessed using paired t-tests. One construct was shortened to assess the model's accuracy at different lengths.

\section{Results}

\subsection{Chemically induced changes in passive mechanics and developed force}

We transformed the measured data from the transverse-force and transverse-deflection IWire coordinate system into longitudinal force and length changes along the construct. This 
allowed us to demonstrate a significant reduction in passive tension after treatment with blebbistatin over all applied transverse loads (Fig. 2A). Our model framework assumed that blebbistatin treatment would abolish the actin-myosin contribution to passive stiffness (represented by $K_{P E a m}$ ), causing the overall passive parameter $K_{M}$ to be equal to $K_{P E n}$ (Eq. (8)). A linear fit to the passive force measurements both before and after introduction of blebbistatin revealed that the non-actin myosin passive stiffness element contributes approximately $67 \%$ percent of the total passive tension in the muscle and about $73 \%$ of the prestress (Fig. 2A). We also measured the effect of isoproterenol treatment, a beta-adrenergic agonist known to increase the rate of calcium cycling and muscle contractility while reducing muscle tension [21]. There was a significant reduction in passive force at every applied load after isoproterenol treatment (Fig. 2B). When analyzing these data using our passive mechanics model, we observed a slight, insignificant decrease in the overall $K_{M}$ as well as a significant reduction of both prestress and $\Delta L_{\text {offset }}$ values (Table 2). We also observed a significant increase in the developed force generated by the constructs (Fig. 2C).

Table 2: Passive mechanic metrics for control and isoproterenol-treated ECTCs

\begin{tabular}{llll}
\hline & Control & Isoproterenol & p-value \\
\hline$K_{M}$ & $0.7 \pm 0.05 \mathrm{Nm}^{-1}$ & $0.6 \pm 0.05 \mathrm{Nm}^{-1}$ & 0.054 \\
prestress & $0.22 \pm 0.02 \mathrm{mN}$ & $0.12 \pm 0.01 \mathrm{mN}$ & $<0.001$ \\
$\Delta L_{\text {offset }}$ & $311 \pm 14 \mu \mathrm{m}$ & $210 \pm 25 \mu \mathrm{m}$ & 0.003 \\
\hline
\end{tabular}

\subsection{Simulation of active contraction}

Once we estimated the passive muscle stiffness, prestress, and offset for each construct, active contraction was simulated at a variety of starting tensions corresponding to the experimental range of stage displacement. An idealized biexponential curve was used to simulate the activation force generated by the CMs in response to electrical stimulation (Fig. 
3A) [8]. We simulated muscle inactivation by setting $K_{S E}$ to zero, and the model predicted no muscle shortening, as was observed with blebbistatin treatment of the constructs. Three active parameters $\left(K_{S E}, K_{\text {PEam }}\right.$, and $\left.b\right)$ were estimated using a nonlinear least squares optimization technique in MATLAB. Regardless of starting conditions or relative elastic element contributions, this procedure consistently found that the isoproterenol-treated constructs had a significant reduction $(50 \% ; p<0.05)$ in the estimated viscosity parameter of the contractile element (b). Independent optimization of $K_{S E}$ and $K_{\text {PEam }}$ also found a significant reduction ( $\mathrm{p}<$ $0.05)$ of $K_{S E}$ in the isoproterenol groups relative to control. In general, this optimization reduced the overall mean squared error of the model fit by between two- and five-fold across the seven constructs. Using these techniques, the model successfully predicted the experimentally measured developed force (Fig. 3B-C) and length changes of individual constructs (Fig. 3D-E). Furthermore, averaging the estimated parameters across constructs gave a robust fit of the average developed force (Fig. 3F).

\subsection{Simulating active contraction with microelectrode trace}

After characterizing the fidelity of our model fit using an idealized biexponential function, we repeated these analyses using data from a microelectrode trace of the action potential as the activation force (Fig. 4A). The estimated model parameters using this alternative activation force function confirmed a reduction of the viscosity parameter $(b)$ with isoproterenol treatment, and the experimental data were well fitted by visual inspection. However, the mean squared error was approximately two- to four-fold higher, relative to that associated with the biexponential activation force function (Fig. 4B-E). Next, we modified the width of the activation force trace to attempt to recreate the mechanical restitution curve described in the companion paper [1]. By varying the action potential duration from $150 \mathrm{~ms}$ to $250 \mathrm{~ms}$ (Fig. 4F), the model

predicted increasing force traces (Fig. 4G) and peak forces (Fig. 4H) that greatly resembled the experimental data presented in Figs. 2 and 4 of the companion paper [1]. 


\subsection{Shortening construct to predict length-related effects}

We tested the model's response to reducing the length of a preformed construct by $\sim 20 \%$ by moving one of the anchoring wires to a new groove in the PDMS mold (Fig. 5A). The experimental results revealed an overall decrease in the peak developed force, as well as a flattening of the downward trend of force generation (Fig. 5B). This experiment was simulated in the model first by assuming that the measured mechanical stiffness of the construct would remain unchanged by the release of tension, merely shifting over to have an offset and prestress of 0 (Fig. $\mathbf{5 C}$, black line). This manipulation resulted in a large mismatch with the experimental passive force data and a poor fit to the peak developed force data (Fig. 5D black line). Next, the $K_{M}$ was estimated using the passive force measurements in the shortened constructs (45\% reduction from original), but prestress was still defined as 0 (Fig. $5 \mathbf{C}$, dark grey line). This correction improved the fit of the passive and developed force (Fig. 5D, dark grey line) but the data were still mismatched. Next, both the $K_{M}$ and prestress were fit to the experimental data, giving a much better fit of the passive force (Fig. 5C, light grey line), but significantly under-predicting the peak force (Fig. 5D, light grey line). Finally, the ratio of $K_{S E}$ to $K_{\text {EEam }}$ was changed to restore the value of the series element stiffness to that of the $K_{S E}$ original construct length, and the results showed significant improvement of developed force fit (Fig. 5D, dashed light grey line), as well as overall fit.

\section{Discussion}

We have presented a model-based method to quantify the passive elasticity and active contractility of an ECTC that is extremely useful to the biomedical, bioengineering, and physiology communities. This analysis allows an in-depth characterization of the mechanics of a 3D cardiac muscle construct that is able to dynamically contract while subjected to a range of applied transverse forces, and it complements the presentation of the data in the companion paper [1]. The analysis strategy presented here allows for translation from force generated in 
the construct applied as a perpendicular load $\left(F_{P}\right)$ to the longitudinal tension internal to and along the length of the construct $\left(F_{C}\right)$. The peak force developed along the construct following contraction consistently decreased with increasing applied tension and applied stretch (Fig. 2C). This was likely a result of the intrinsic force-length relationship of striated muscle cells, including CMs, in which the force-generating potential rapidly declines past the optimal length [5;22]. This construct-focused analysis also makes it possible to estimate the prestress generated by CMs as well as fibroblasts during the process of construct formation and compaction. We demonstrated that this stress is significantly reduced by both blebbistatin and isoproterenol treatments. Blebbistatin is known to disrupt actin/myosin interactions within CM sarcomeres and has been shown to disrupt traction forces generated by fibroblasts [23;24].

This model also enables simulation of active muscle contraction. The comparisons in Figs. 3 and 4 demonstrate that either a biexponential curve or that resembling an action potential can be used as activation force inputs to drive the model, although the biexponential activation force trace delivers consistently better fits to the experimental measurements. In the future, microelectrode trace measurements could be used to predict altered mechanics in response to genetic mutations or drug effects. Parameter estimation using either activation force function revealed a significant decrease in effective contractile element viscosity $(b)$, and suggests a potential decrease in $K_{S E}$ after treatment with isoproterenol. Both effects are likely related to the increase in calcium availability and turnover mediated by isoproterenol, which decreases the short-term stiffening effect of actin-myosin interactions $[11 ; 21 ; 25]$. Future experiments to image intracellular calcium transients during contraction should make it possible to quantify these changes.

The collection of experimental data examined here was not sufficient to definitively differentiate the relative contributions of the model parameters and the active force function using the nonlinear least squares estimation method. The dynamic nature of the modified Hill 
model, however, allows prediction of mechanical responses to differing mechanical and electrical stimuli. Thus, it is possible to consider hypothetical experiments in silico, and to design experiments that are more highly informative about the underlying model components. For example, in order to distinguish the model parameters from the active force function, a natural next step experimentally would be to vary the rate and shape of mechanical loading in the absence of electrical stimuli (i.e., no active force). Supplementary Fig. S6 illustrates the mechanical stimuli and resulting passive changes in construct length for two such hypothetical experiments. These findings would permit independent assessment of the contributions of $K_{\text {PEam }}$, $K_{S E}$ and $b$, which in turn would enable an inverse analysis of the data to estimate the activation force traces. Further development of the model should benefit from additional measurements that record the calcium transients associated with $\mathrm{CM}$ contraction and that image the differences in both transmembrane calcium potential between simultaneous field stimulation of the entire construct and the response that propagates from a bipolar stimulating electrode at one end of the fiber. It is likely that these calcium traces will correspond with the predicted activation force traces, improving the model fit and providing insight into electromechanical coupling.

This model is also able to recreate changes in muscle mechanics in response to changing electrical and physical inputs. Recreating the pacing frequency restitution curve confirms that using action potential traces is a viable input for predicting contractile response. Furthermore, the shortening experiments provided an interesting opportunity to test and refine the model. The peak force developed by the shortened ECTC was significantly lower than that for the original length, which suggests that the construct length has been reduced below some optimal length, thus reducing force-generating capacity. Our initial assumption that construct stiffness would be preserved with a large reduction in length proved to be a poor fit to the data. Subsequent trials revealed that at the reduced length, the construct has a much lower linear passive stiffness and 
retains some prestress even at low initial stretch. These results indicate that the stiffness which appeared linear at the original length is actually part of an exponential or partially nonlinear stiffness function common in biological tissues [11;26].

The I-Wire platform and these measurements and estimates of intrinsic muscle properties $\left(K_{M}\right.$, prestress, and viscosity $\left.b\right)$ provide a powerful new tool for quantifying the effects of pharmacological strategies on $\mathrm{CM}$ function in vitro. Of particular importance, the constructs have a uniform cross-sectional area over most of their length, in contrast to animal papillary and trabecular muscles. In addition, it is feasible to study 3D ECTCs formed with CMs that are derived from human induced pluripotent stem cells. The I-Wire platform and our model-based analysis will also inform more complex models of heart function for interventional planning purposes $[10 ; 12-14 ; 27-30]$.

With the advancement of induced pluripotent stem cell (iPSC) technologies specific to the heart [31;32], there is the possibility of being able to use cell-based technologies to support cardiac regeneration $[33 ; 34]$. This would then present the challenge of qualifying the cells that will be used to repair an ailing heart - one must be able to assess the mechanical, electrical, and metabolic potential of the cells prior to their use in a patient, particularly if those cells were derived from the patient's own iPSCs. The I-Wire platform in the accompanying paper [1] in combination with the modeling described in this one may provide this capability: prior to the utilization of the cardiomyocytes, fibroblasts, and other cells for regenerative therapy, these same cells could be used to create an in vitro I-Wire construct whose performance could be evaluated using a standardized protocol. Given such a protocol, one then might be able to further refine the process by which these cells are derived to better optimize their performance in vivo.

Overall, we believe that this new technique will improve our understanding of the complex interplay between CM mechanics and function, and will also serve as a demonstration of model- 
based quantification of mechanically active tissue equivalents. Furthermore, we have in this paper outlined a computational procedure that should be applicable to many other active, contractile biomaterials and their mimics [35-37].

\section{Acknowledgements}

The authors were supported in part by the National Institutes of Health (1R01118392-01), the National Science Foundation (1055384 and DGE-0909667), the American Heart Association (15PRE25710333), and the Vanderbilt Institute for Integrative Biosystems Research and

Education. The content is solely the responsibility of the authors and does not necessarily represent the official views of the funding agencies. The authors thank Allison Price and Jeffrey Davidson for their editorial comments on this manuscript.

\section{Disclosures}

The authors have declared that no conflicts of interest exist.

\section{Appendix A. Supplementary Material}

Supplementary Material related to this article can be found at

\section{Figure Captions}

Fig. 1. Platform description and model schematic. (A) Representation of the I-Wire construct from above, with the unloaded position of the probe indicated by the dotted green circle. (B) Image of I-Wire construct. (C) Schematic simplified to highlight relevant measurements. See text for variable names. (D) Diagram of the relevant mechanical model (a modified, Hill type model), consisting of the muscle mechanics components (left side of dashed line) linked to a nonlinear (with respect to $L$ ) spring representing the probe (right side of dashed line).

Fig. 2. Passive and developed forces in constructs are affected by pharmacological perturbations. (A) Passive force in constructs treated with blebbistatin was significantly reduced 
$(p<0.01)$ for all samples. (B) Passive force was significantly reduced $(p<0.01)$ between all isoproterenol treated samples, and the predicted and calculated force in the ECTC had relatively close agreement with experimental data. (C) Peak developed force was significantly increased $(p<0.05)$ in the isoproterenol-treated constructs at most applied transverse loads (imposed by stage displacement that deflects the probe) ( ${ }^{*}$ indicates $p<0.5$ ). Experimental data presented as mean \pm SEM.

Fig. 3. Predicted active force in ECTCs. (A) Biexponential activation force input used. (B,C) Predicted developed force and $(D, E)$ relative predicted length compared to measured data for representative construct. (F) Predicted model fit to average data using average of best fit parameters. Experimental data presented as mean \pm SEM.

Fig. 4. Simulation of active contraction using construct action potential. (A) Activation force shape taken from a measurement of the action potential voltage (1) scaled similarly to idealized biexponential function. (B) Developed force traces, $(C)$ peak forces, and $(D, E)$ relative length fit in a representative construct. (F) Horizontal scaling of activation force traces to match measured values at different pacing frequencies. $(G)$ Predicted model response to these inputs for the construct at a simulation stage position of $1400 \mu \mathrm{m}$, and $(\mathrm{H})$ the peak developed force plotted over the estimated pacing frequency.

Fig. 5. Shortening construct to predict length-related effects. (A) Schematic of construct shortening. (B) Traces of developed force in the original and shortened configurations. The thicker lines represent the shortened configuration. (C,D) Experimental and model fits of passive tension and peak developed force in construct before and after shortening.

\section{References}

[1] V.Y. Sidorov, P.C. Samson, T.N. Sidorova, J.M. Davidson, C.C. Lim, J.P. Wikswo, I-Wire Heart-on-a-Chip I: Three-dimensional cardiac tissue constructs for physiology and pharmacology, Acta Biomater. (submitted) (2016). 
[2] A.V. Hill, The Heat of Shortening and the Dynamic Constants of Muscle, Proc.R.Soc.Lond.B 126 (1938) 136-195.

[3] N. Milani-Nejad, Y. Xu, J.P. Davis, K.S. Campbell, P.M.L. Janssen, Effect of muscle length on cross-bridge kinetics in intact cardiac trabeculae at body temperature, J.Gen.Physiol. 141 (2013) 133-139.

[4] H.A. Shiels, E. White, The Frank-Starling mechanism in vertebrate cardiac myocytes, J.Exp.Biol. 211 (2008) 2005-2013.

[5] A.V. Hill, First and Last Experiments in Muscle Mechanics, Cambridge University Press, London, 1970.

[6] A.V. Hill, The Mechanics of Active Muscle, Proc.R.Soc.Lond.B 141 (1953) 104-117.

[7] T.A. McMahon, Muscles, Reflexes, and Locomotion, Princeton University Press, Princeton, NJ, 1984.

[8] R.W.S. Shadmehr, A Simple Muscle Model (Supplementary Document), The computational neurobiology of reaching and pointing: a foundation for motor learning, MIT Press, Cambridge, MA, 2005.

[9] D. Chapelle, F. Clement, F. Genot, P.L. Tallec, M. Sorine, J.M. Urquiza, A PhysiologicallyBased Model for the Active Cardiac Muscle Contraction, in: T. Katila, J. Nenonen, I. E. Magnin, P. Clarysse, J. Montagnat (Eds.), Functional Imaging and Modeling of the Heart, Springer, Berlin, Heidelberg, 2001, pp. 128-133.

[10] M. Sermesant, P. Moireau, O. Camara, J. Sainte-Marie, R. Andriantsimiavona, R. Cimrman, D.L.G. Hill, D. Chapelle, R. Razavi, Cardiac function estimation from MRI using a heart model and data assimilation: Advances and difficulties, Med.Image.Anal. 10 (2006) 642-656.

[11] J.M. Winters, Hill-Based Muscle Models: A Systems Engineering Perspective, in: J. M. Winters, S. L. Y. Woo (Eds.), Multiple Muscle Systems: Biomechanics and Movement Organization, Springer, New York, NY, 1990, pp. 69-93.

[12] K.D. Costa, J.W. Holmes, A.D. McCulloch, Modelling cardiac mechanical properties in three dimensions, Philos.Trans.R.Soc.London A 359 (2001) 1233-1250.

[13] V.Y. Wang, H.I. Lam, D.B. Ennis, B.R. Cowan, A.A. Young, M.P. Nash, Modelling passive diastolic mechanics with quantitative MRI of cardiac structure and function, Med.Image.Anal. 13 (2009) 773-784.

[14] K.L. Sack, N.H. Davies, J.M. Guccione, T. Franz, Personalised computational cardiology: Patient-specific modelling in cardiac mechanics and biomaterial injection therapies for myocardial infarction, Heart Fail.Rev. doi: 10.1007/s10741-016-9528-9 (2016).

[15] V.Y. Sidorov, M.C. Woods, J.P. Wikswo, Effects of elevated extracellular potassium on the stimulation mechanism of diastolic cardiac tissue, Biophys.J. 84 (2003) 3470-3479. 
[16] A.F. Straight, A. Cheung, J. Limouze, I. Chen, N.J. Westwood, J.R. Sellers, T.J. Mitchison, Dissecting temporal and spatial control of cytokinesis with a myosin II inhibitor, Science 299 (2003) 1743-1747.

[17] M. Kovacs, J. Toth, C. Hetenyi, A. Malnasi-Csizmadia, J.R. Sellers, Mechanism of blebbistatin inhibition of myosin II, J.Biol.Chem. 279 (2004) 35557-35563.

[18] O.E. Brodde, M.C. Michel, Adrenergic and Muscarinic Receptors in the Human Heart, Pharmacol.Rev. 51 (1999) 651-690.

[19] L.B. Hazeltine, C.S. Simmons, M.R. Salick, X. Lian, M.G. Badur, W. Han, S.M. Delgado, T. Wakatsuki, W.C. Crone, B.L. Pruitt, S.P. Palecek, Effects of substrate mechanics on contractility of cardiomyocytes generated from human pluripotent stem cells, Int.J.Cell Biol. 2012 (2012) Article 508294.

[20] V.V. Fedorov, S. Leonov, Optimal design for nonlinear response models, CRC Press, Boca Raton, FL, 2013.

[21] I. Suard, N. Pery-Man, C. Coirault, J.C. Pourny, Y. Lecarpentier, D. Chemla, Relaxant effects of isoproterenol in isolated cardiac muscle: influence of loading patterns, Am.J.Physiol. 267 (1994) H1814-H1823.

[22] K.B. Campbell, M. Chandra, R.D. Kirkpatrick, B.K. Slinker, W.C. Hunter, Interpreting cardiac muscle force-length dynamics using a novel functional model, Am.J.Physiol.Heart 286 (2004) H1535-H1545.

[23] K.A. Beningo, K. Hamao, M. Dembo, Y.L. Wang, H. Hosoya, Traction forces of fibroblasts are regulated by the Rho-dependent kinase but not by the myosin light chain kinase, Arch.Biochem.Biophys. 456 (2006) 224-231.

[24] J.S. Allingham, R. Smith, I. Rayment, The structural basis of blebbistatin inhibition and specificity for myosin II, Nat.Struct.Mol.Biol. 12 (2005) 378-379.

[25] F.I. Malik, J.J. Hartman, K.A. Elias, B.P. Morgan, H. Rodriguez, K. Brejc, R.L. Anderson, S.H. Sueoka, K.H. Lee, J.T. Finer, R. Sakowicz, R. Baliga, D.R. Cox, M. Garard, G. Godinez, R. Kawas, E. Kraynack, D. Lenzi, P.P. Lu, A. Muci, C.R. Niu, X.P. Qian, D.W. Pierce, M. Pokrovskii, I. Suehiro, S. Sylvester, T. Tochimoto, C. Valdez, W.Y. Wang, T. Katori, D.A. Kass, Y.T. Shen, S.F. Vatner, D.J. Morgans, Cardiac Myosin Activation: A Potential Therapeutic Approach for Systolic Heart Failure, Science 331 (2011) 1439-1443.

[26] E.S. Grood, R.E. Mates, H. Falsetti, Model of Cardiac-Muscle Dynamics, Circ.Res. 35 (1974) 184-196.

[27] L.C. Lee, Z.H. Zhang, A. Hinson, J.M. Guccione, Reduction in Left Ventricular Wall Stress and Improvement in Function in Failing Hearts using Algisyl-LVR, J.Vis.Exp.2013) Article UNSP e50096.

[28] J.F. Wenk, L. Ge, Z.H. Zhang, D. Mojsejenko, D.D. Potter, E.E. Tseng, J.M. Guccione, M.B. Ratcliffe, Biventricular Finite Element Modeling of the Acorn CorCap Cardiac Support Device on a Failing Heart, Ann.Thorac.Surg. 95 (2013) 2022-2027. 
[29] J.F. Wenk, L. Ge, Z.H. Zhang, M. Soleimani, D.D. Potter, A.W. Wallace, E. Tseng, M.B. Ratcliffe, J.M. Guccione, A coupled biventricular finite element and lumped-parameter circulatory system model of heart failure, Comput.Methods Biomech.Biomed.Eng. 16 (2013) 807-818.

[30] J.F. Wenk, K. Sun, Z.H. Zhang, M. Soleimani, L.A. Ge, D. Saloner, A.W. Wallace, M.B. Ratcliffe, J.M. Guccione, Regional Left Ventricular Myocardial Contractility and Stress in a Finite Element Model of Posterobasal Myocardial Infarction, J.Biomech.Eng.- T.ASME 133 (2011) Article 044501.

[31] M. Fujiwara, P.S. Yan, T.G. Otsuji, G. Narazaki, H. Uosaki, H. Fukushima, K. Kuwahara, M. Harada, H. Matsuda, S. Matsuoka, K. Okita, K. Takahashi, M. Nakagawa, T. Ikeda, R. Sakata, C.L. Mummery, N. Nakatsuji, S. Yamanaka, K. Nakao, J.K. Yamashita, Induction and Enhancement of Cardiac Cell Differentiation from Mouse and Human Induced Pluripotent Stem Cells with Cyclosporin-A, PloS.One. 6 (2011) Article e16734.

[32] D.J. Gauthier, Resource Letter: CC-1: Controlling chaos, Am.J.Phys. 71 (2003) 750-759.

[33] G. Vunjak-Novakovic, K.O. Lui, N. Tandon, K.R. Chien, Bioengineering Heart Muscle: A Paradigm for Regenerative Medicine, Annu.Rev.Biomed.Engr. 13 (2011) 245-267.

[34] M.A. Laflamme, C.E. Murry, Heart regeneration, Nature 473 (2011) 326-335.

[35] H. Stoyanov, M. Kollosche, S. Risse, R. Wache, G. Kofod, Soft Conductive Elastomer Materials for Stretchable Electronics and Voltage Controlled Artificial Muscles, Adv.Mater. 25 (2013) 578-583.

[36] M.M. Ma, L. Guo, D.G. Anderson, R. Langer, Bio-Inspired Polymer Composite Actuator and Generator Driven by Water Gradients, Science 339 (2013) 186-189.

[37] B.K. Juluri, A.S. Kumar, Y. Liu, T. Ye, Y.W. Yang, A.H. Flood, L. Fang, J.F. Stoddart, P.S. Weiss, T.J. Huang, A Mechanical Actuator Driven Electrochemically by Artificial Molecular Muscles, Acs Nano 3 (2009) 291-300. 
A

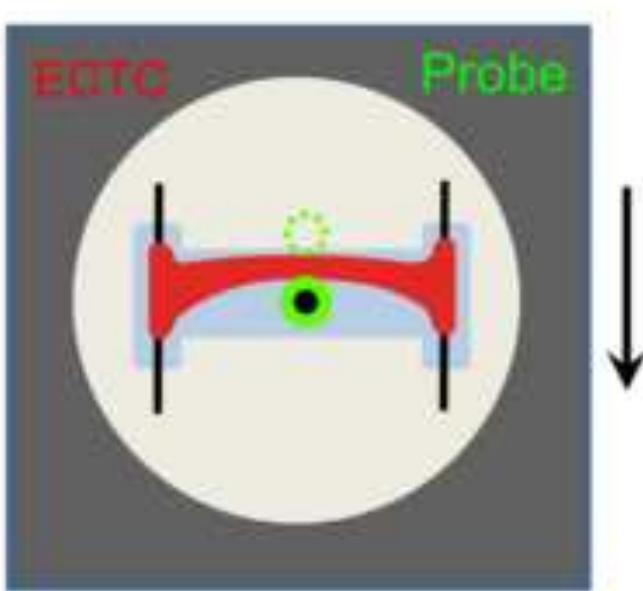

B

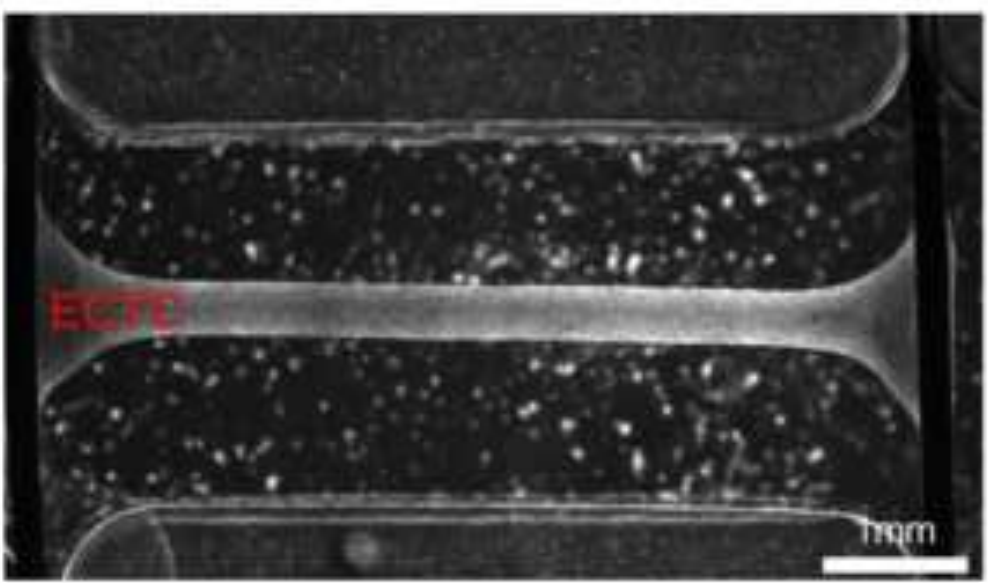

$\mathrm{hmm}$

c

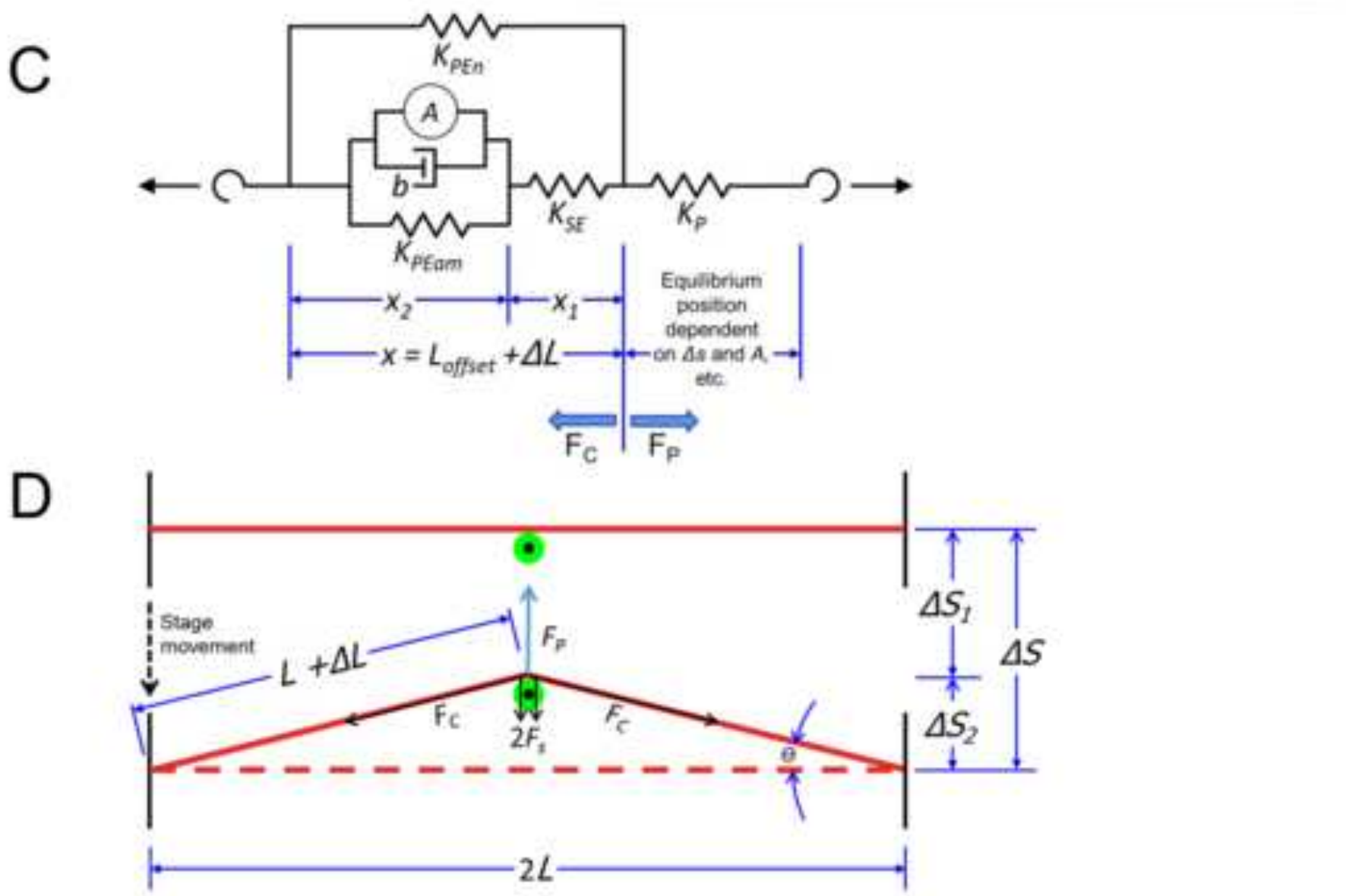

D

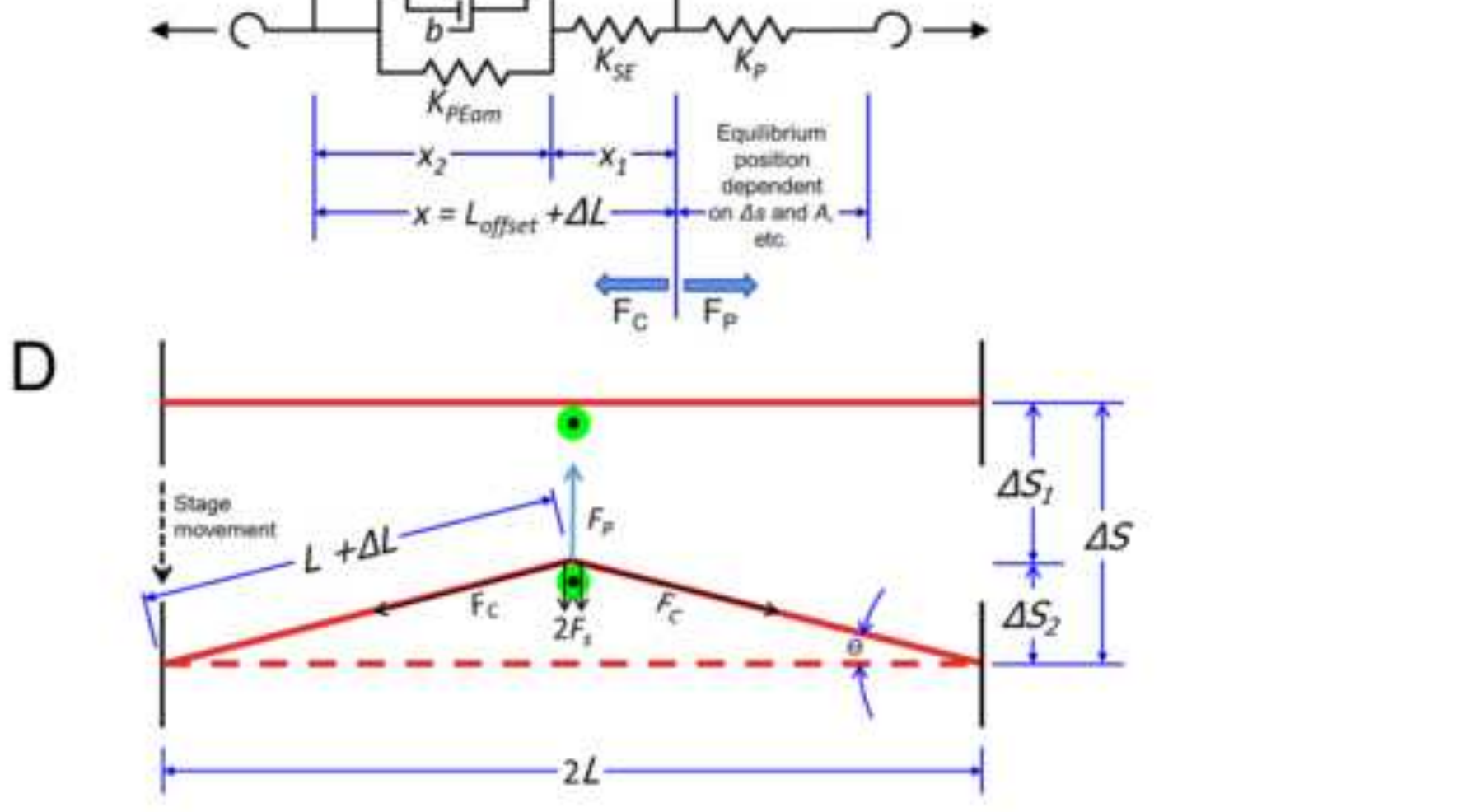

Stage

movement

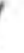

( 


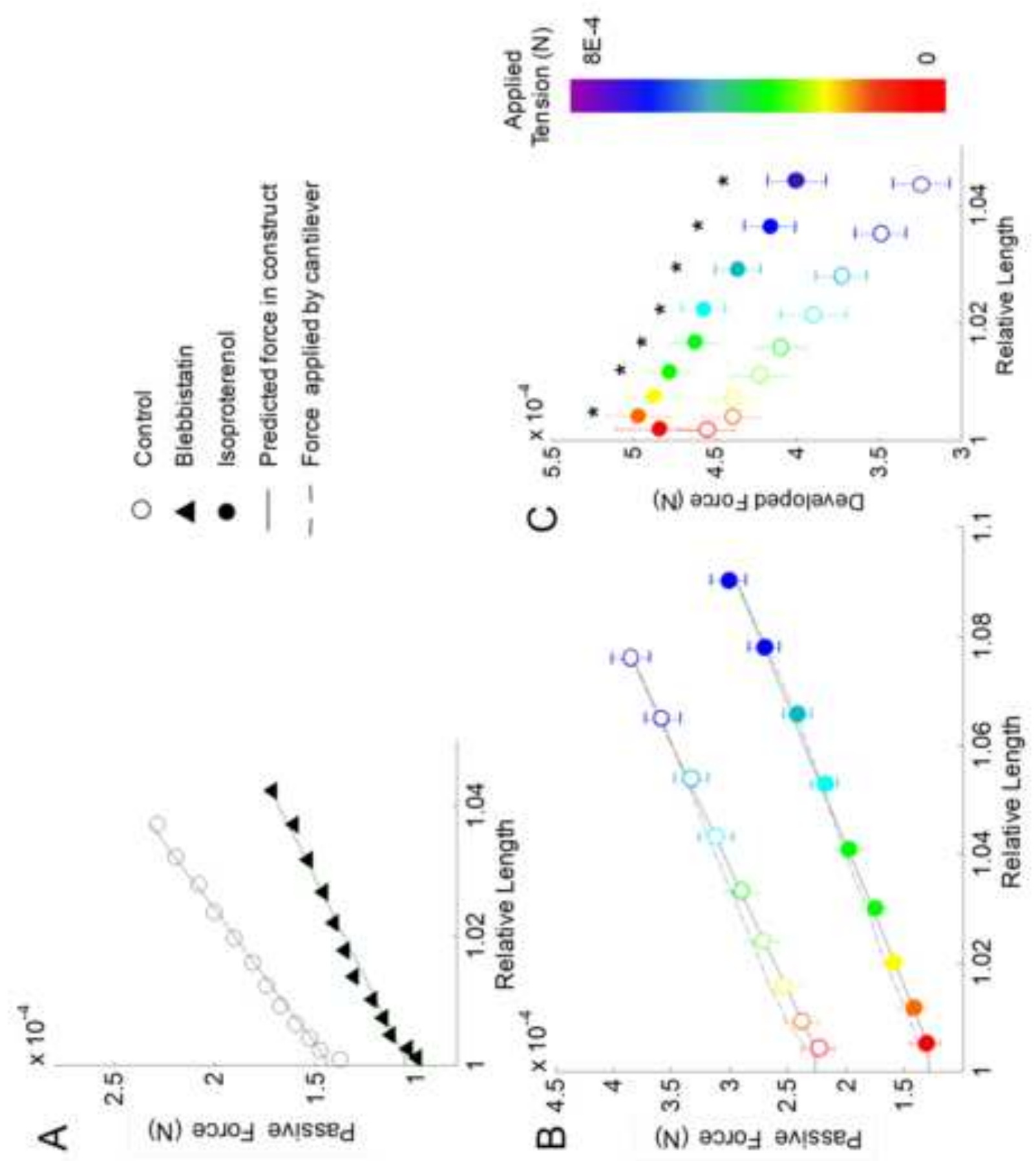



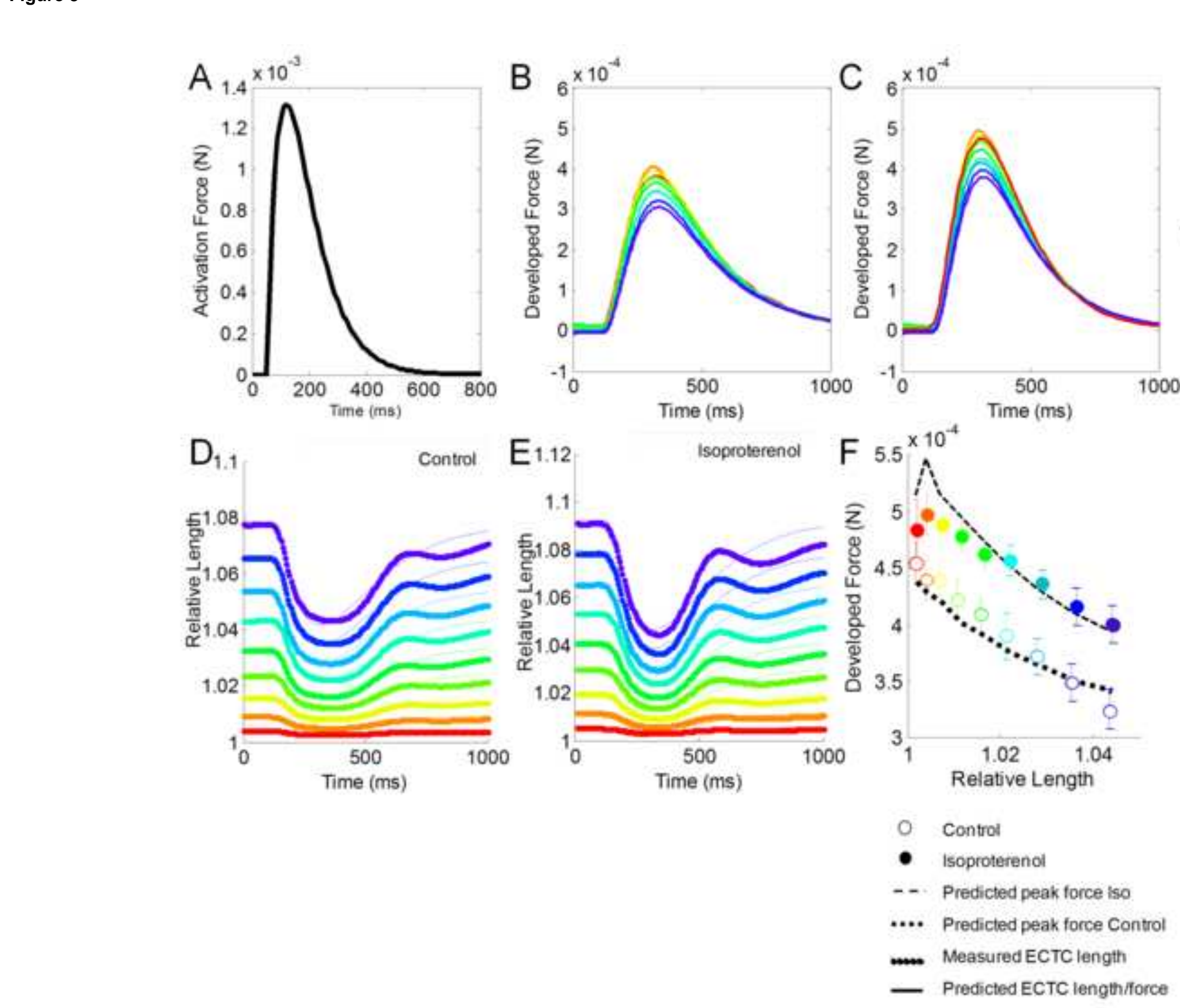

Applied Tension $(\mathrm{N})$
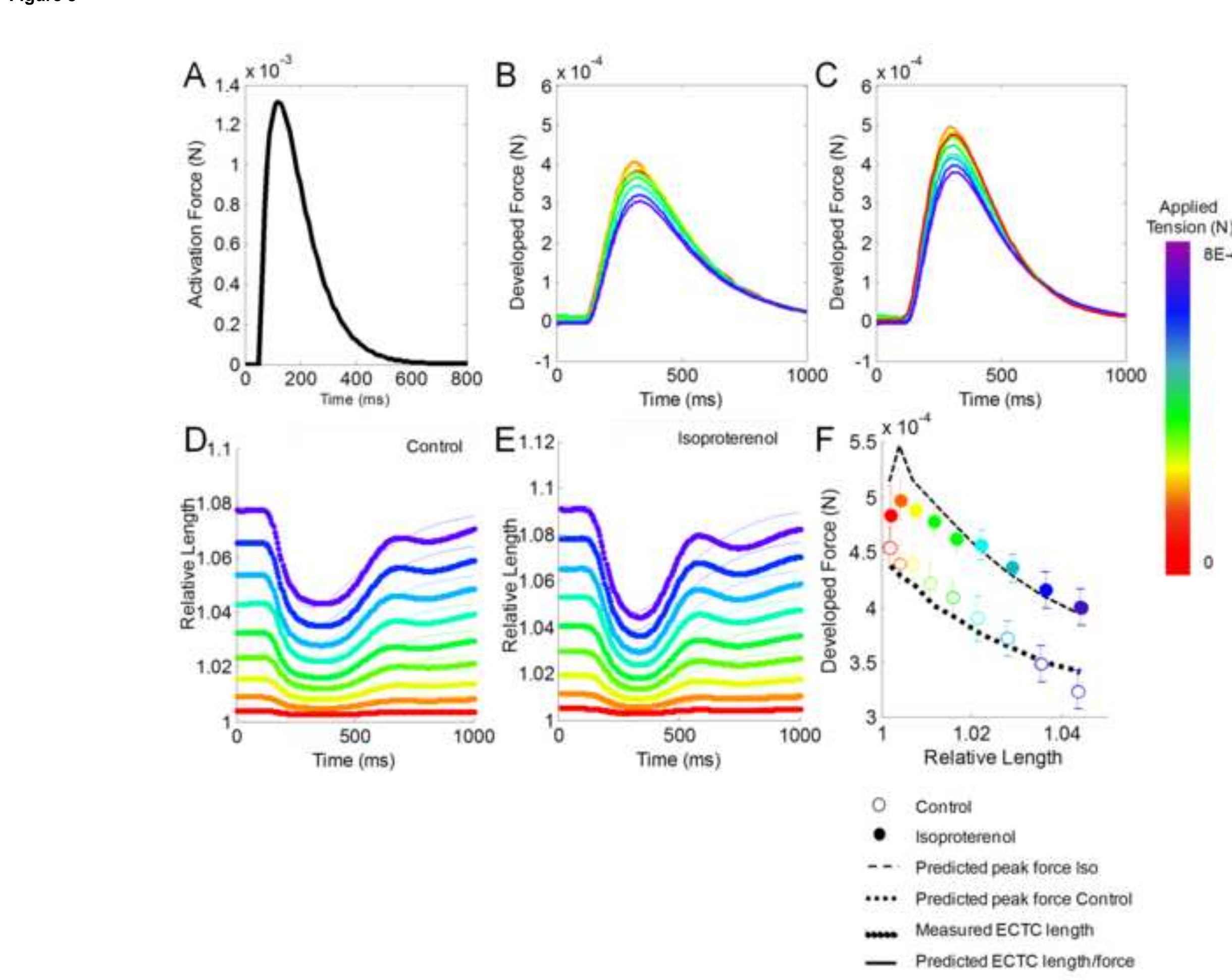

- Control

- isoproterenol

- Predicted peak force iso

.... Predicted peak force Control

men Measured ECTC length

- Predicted ECTC length/force 

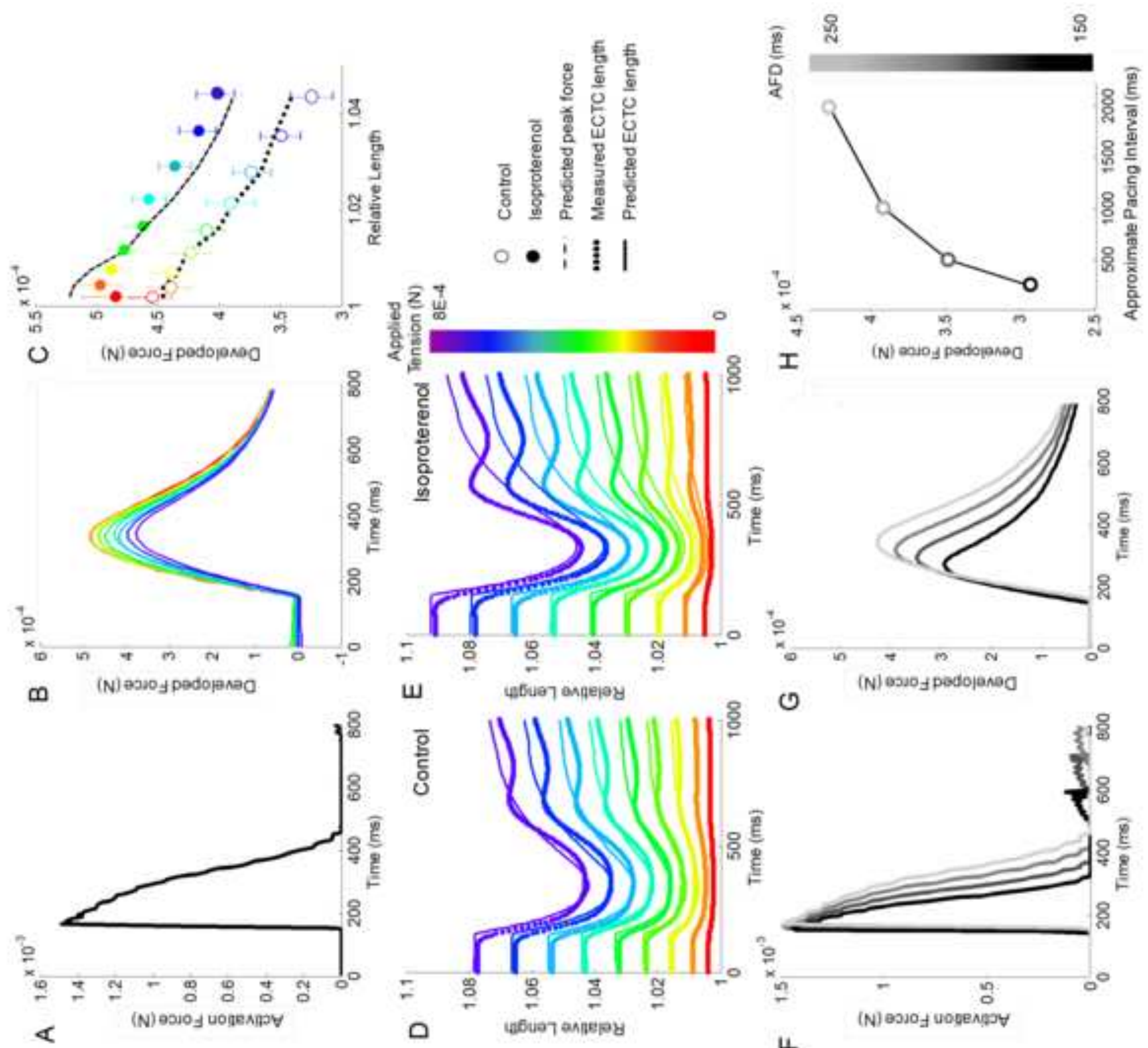

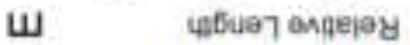
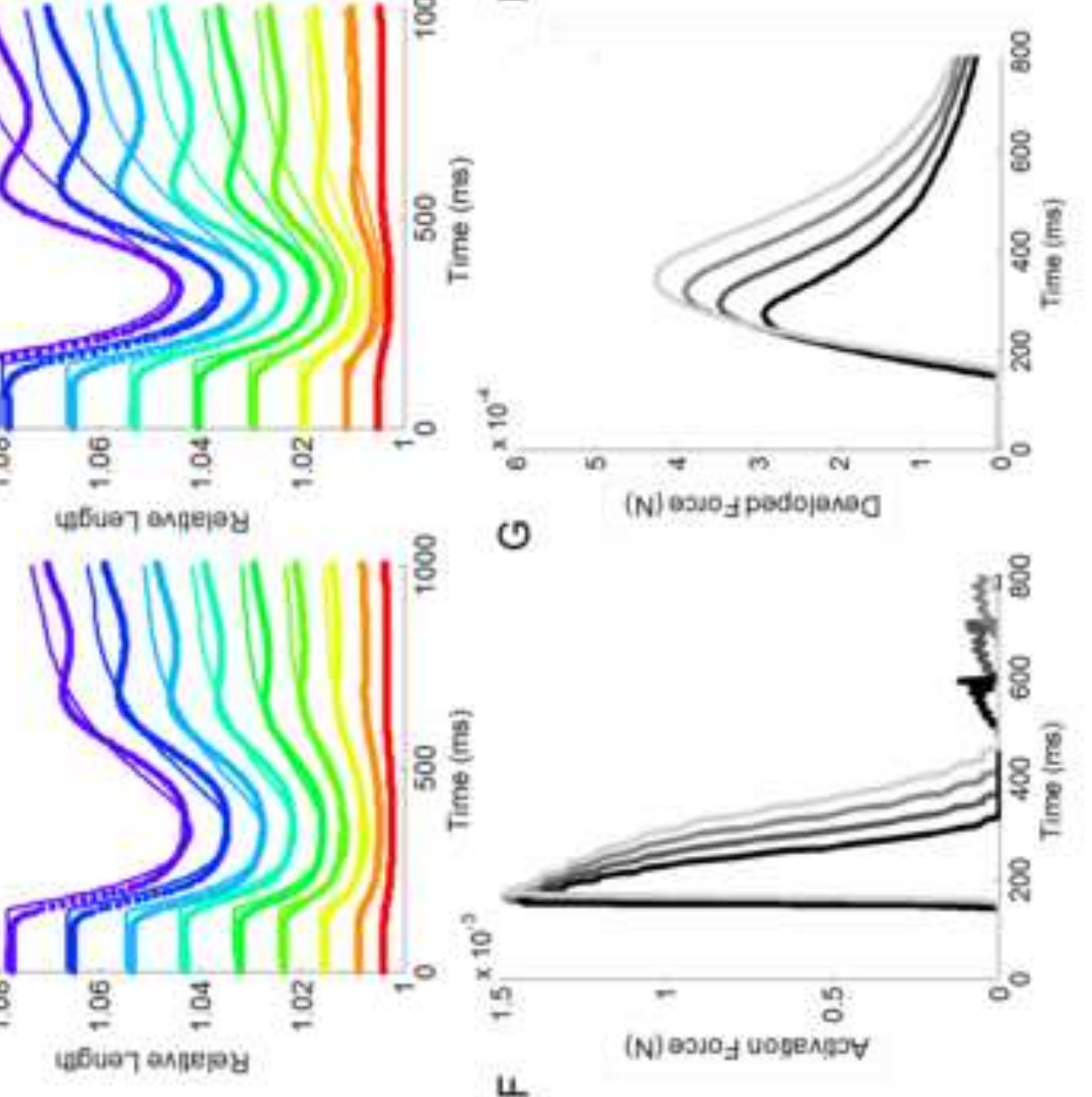

$\circ$

(N) esuog padojanad

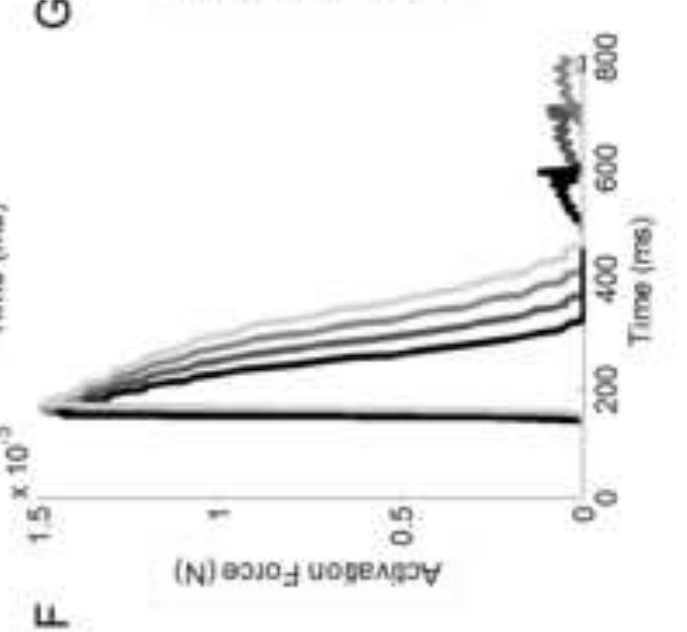

4

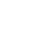




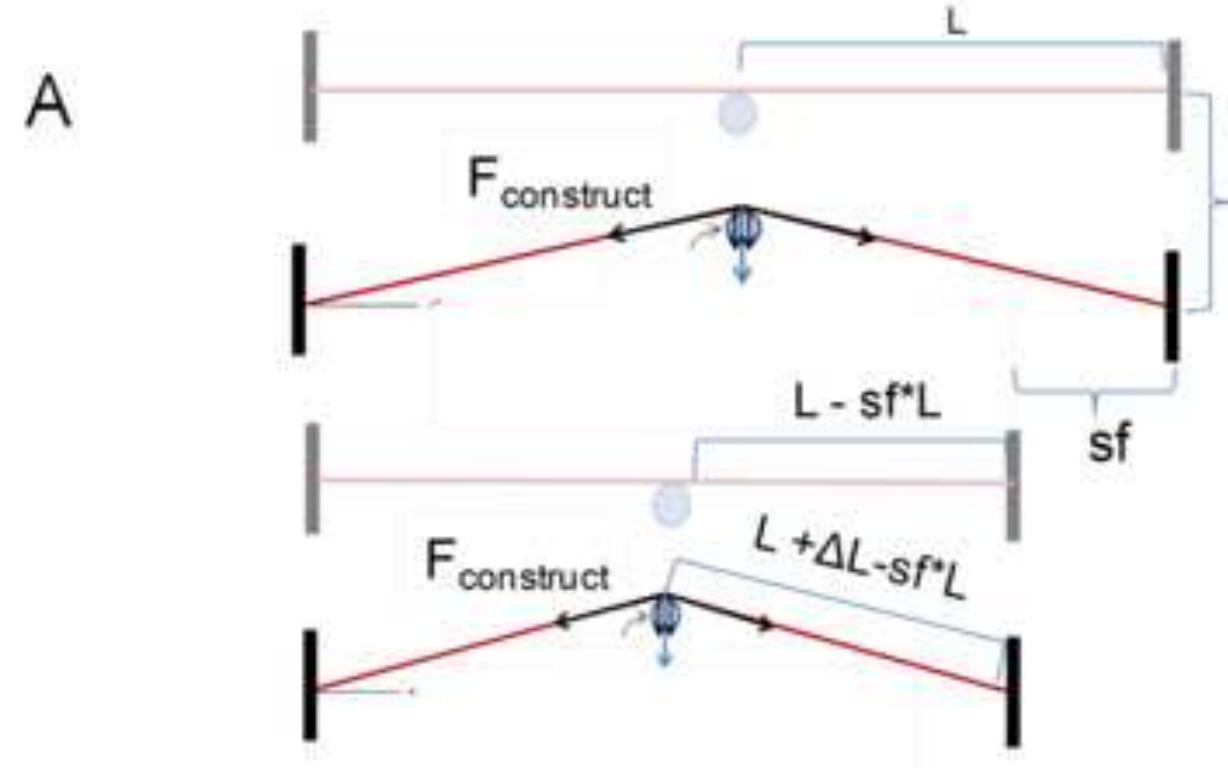

\section{Stage movement}

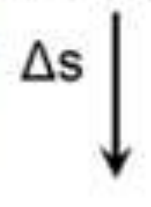

- Control

- Shortened

- Predicted ECTC force, initial

- Predicted ECTC force after shortening

- Predicted ECTC force with fit $K_{M}$

- Predicted ECTC force with fit $K_{M}$ and prestress

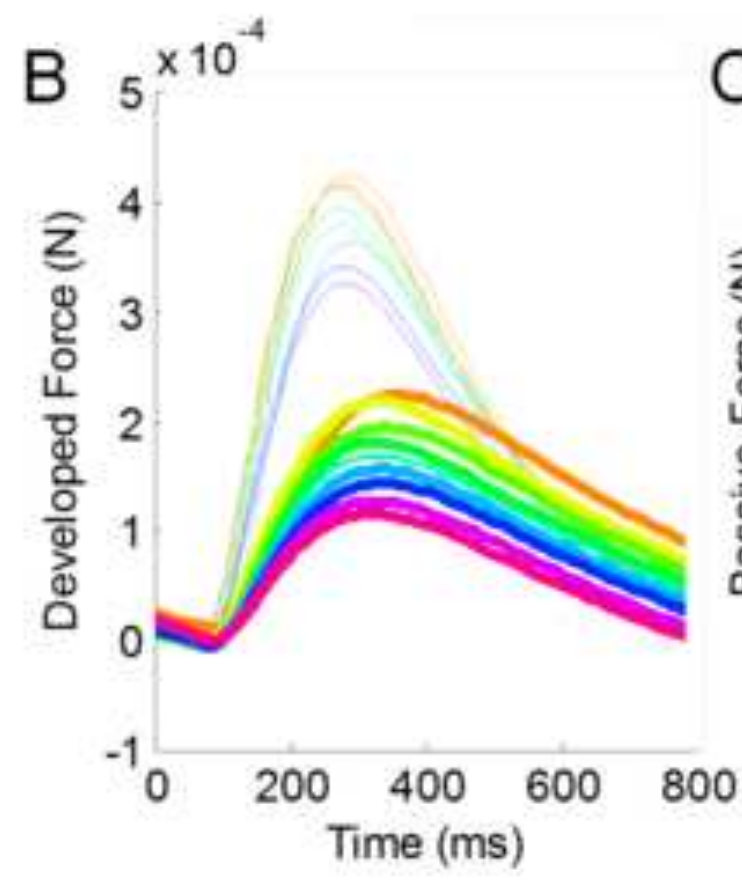

C $_{6} \times 10^{-4}$

D ${ }_{5} \times 10^{-4}$

Applied Tension $(\mathrm{N})$
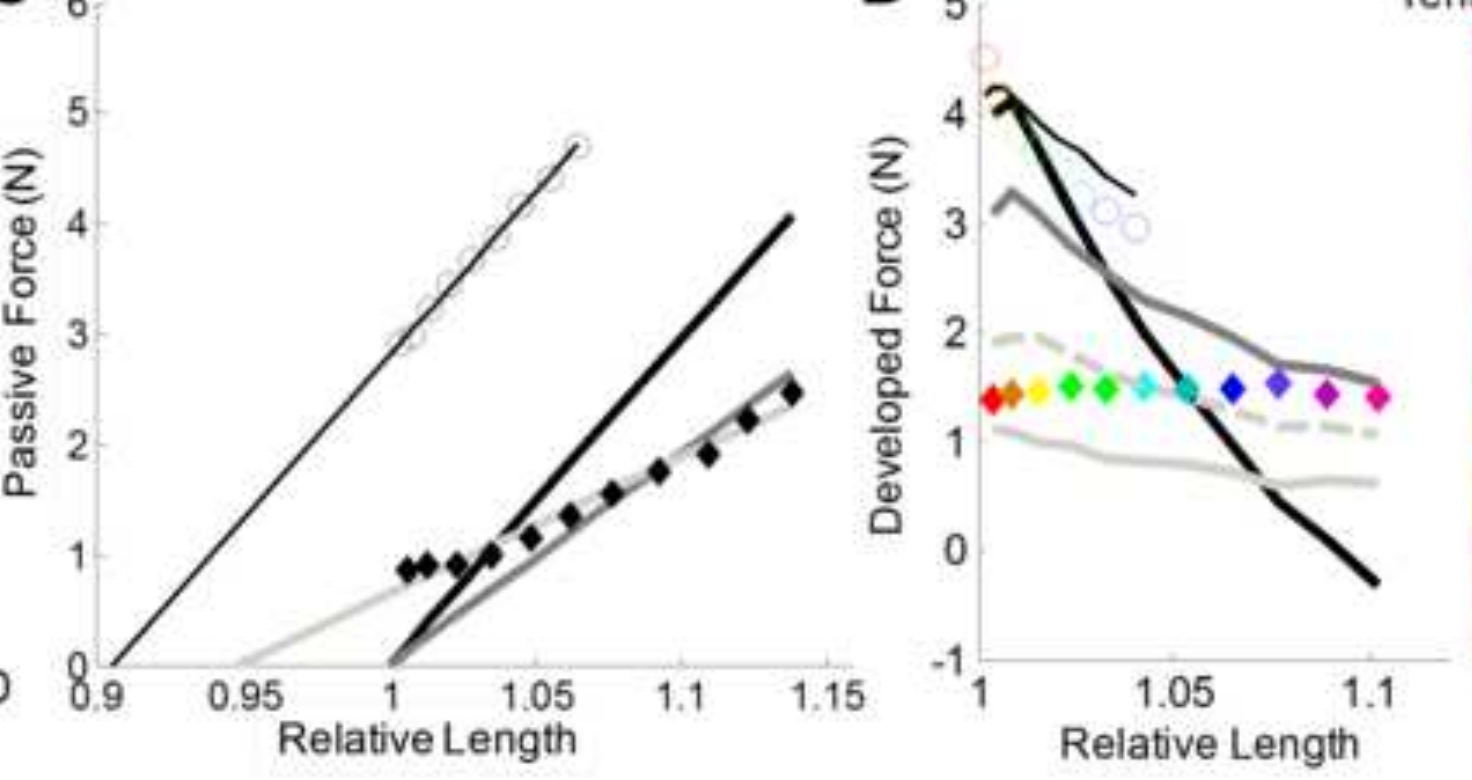

$1 \mathrm{E}-3$ 


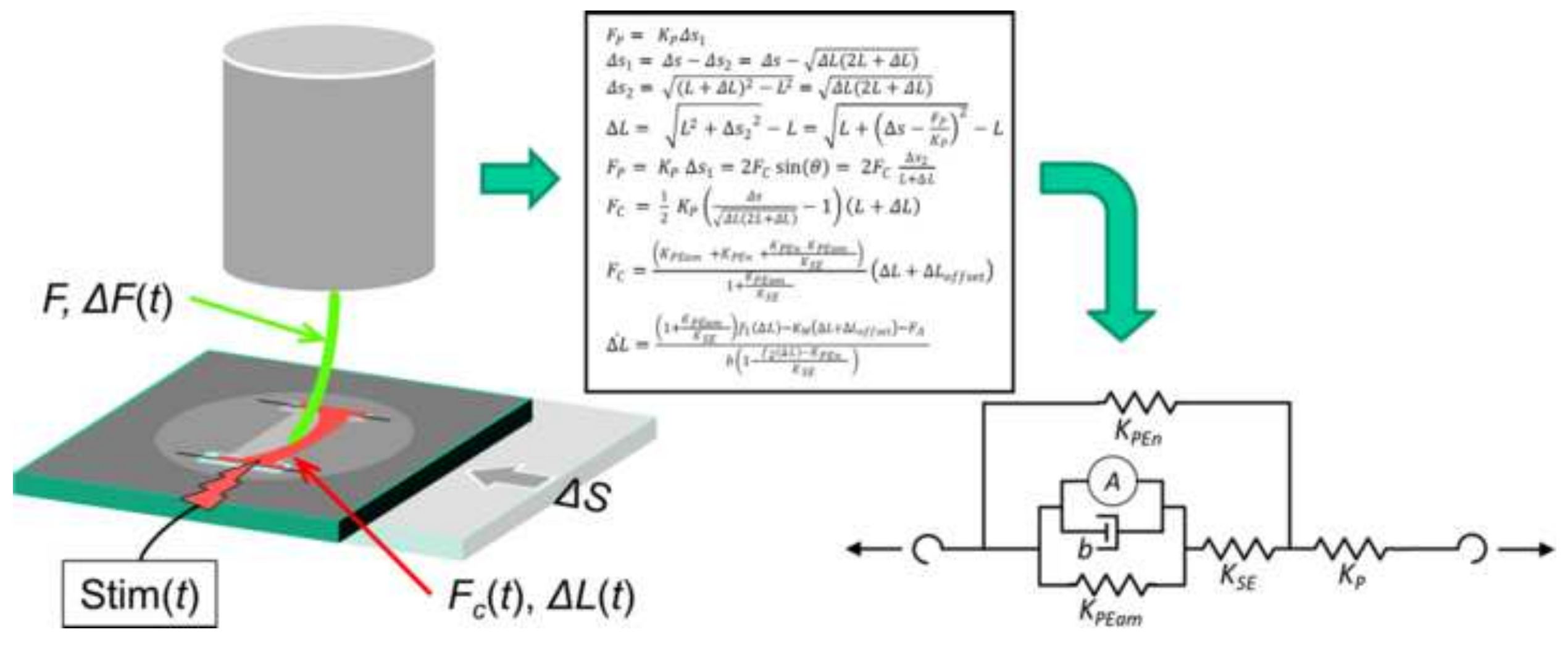

